\title{
HMRP: Hierarchy-Based Multipath Routing Protocol for Wireless Sensor Networks
}

\author{
Ying-Hong Wang ${ }^{1}$, Hung-Jen Mao ${ }^{1}$, Chih-Hsiao Tsai ${ }^{1}$, and Chih-Chieh Chuang ${ }^{2}$ \\ ${ }^{1}$ Department of Computer Science and Information Engineering, \\ TamKang University Tamsui, Taipei, Taiwan, R.O.C \\ inhon@mail.tku.edu.tw, 693192238@s93.tku.edu.tw, \\ 890190092@s90.tku.edu. tw \\ ${ }^{2}$ Computer and Communications Research Laboratories, \\ Industrial Technology Research Institute, Tainan City, Taiwan, R.O.C \\ twjack@itri.org.tw
}

\begin{abstract}
Wireless sensor networks consist of small nodes with sensing, computation, and wireless communications capabilities. The energy efficiency is a key design issue that needs to be enhanced to improve the life span of the network. In this paper, we propose a Hierarchy-Based Multipath Routing Protocol (HMRP) for wireless sensor networks. In HMRP, the network will be constructed to layered-network at first. Based on the layered-network, sensor nodes will have multipath route to sink node through some candidate parent nodes. The simulation results show that our HMRP can improve the lifetime of sensor networks.
\end{abstract}

\section{Introduction}

Wireless sensor networks consist of hundreds to thousands of sensor nodes that have low-power, processor, and limited memory and radio equipments. Those multifunction sensor nodes will be employed in a wide range of applications. Such like military, battlefield, environment monitoring, and civil all can be applied widely with sensor network. Recently, this technology is developed more quickly because of its amount of costs.

The sensor nodes are responsible for collecting information and returning the data it sensed to the Base Station (or Sink node). An important challenge in the design of these networks is battery energy, which limit the lifetime and quality of the networks. Therefore, in order to prolong the lifetime of sensor networks, it is important to design good routing protocols for wireless sensor networks. In [4, 5], the placement of classical sensors will be predetermined and the topology of the network will be in advance. However, in the case of those methods, sensor nodes on the routing path will deplete their energy very fast because of these fixed paths to transfer the sensed data back to the base station (BS). In the past, sensor nodes forward the data packet to the BS directly. But those sensor nodes will consume their battery quickly, so there are many multihop routing protocols proposed in [1, 3, 6, 7, 8, 9] to forward the data packet back to the BS through other nodes. And hierarchical technologies are proposed in $[2,7,8,9]$. Because sensor nodes will spend a lot of energies when transmit 
and receive messages in wireless sensor networks. Hence, the hierarchical routing is an efficient way to decrease energy consumption with data aggregation and fusion.

In this paper, we present an energy-efficient hierarchical mechanism, termed Hierarchy-Based Multipath Routing Protocol (HMRP). We have designed HMRP with the following goals in mind:

Scalability. Since unconstrained scale is an inherent feature of sensor network, the solution has to scale to small or large network size.

Simplicity. The sensors have limited computing capability and memory resources; we seek to minimize the number of operation performed and the states maintained at each node.

System Lifetime. These networks could function for as long as possible. It may be inconvenient or impossible to recharge node batteries. Therefore, all aspects of the node, from the hardware to the protocols, must be designed to be extremely energy efficient.

This paper is organized as follows. Section 2 explains some related technicalities. Section 3 we propose a hierarchy-Based multipath routing protocol for wireless sensor networks. Next, section 4 we present the simulation results. Finally, We give the conclusions in section 5 .

\section{Related Works}

In this section we will introduce several hierarchical protocols. A clustering scheme called Low-Energy Adaptive Clustering Hierarchy (LEACH) is proposed in [2] that employs the technique of randomly rotating the role of a cluster head among all the nodes in the network. The operation of LEACH is organized in rounds where each round consists of a setup phase and a transmission phase. During the setup phase, the network will be separated some clusters and will select a cluster head in each cluster randomly. During the transmission phase, the cluster heads collect data from nodes within their respective clusters and apply data fusion before forwarding them directly to the BS. LEACH provides sensor networks with many good features, such as clustering-based, two roles of sensor nodes. However, it expenses much energy in cluster heads when forward data packets to the BS directly.

Another clustering-based routing protocol is Power Efficient Gathering in Sensor Information Systems (PEGASIS) [1], which is constructed from chain-based by using a greedy algorithm. Each node transmits to and receives from only one of its neighbors. In each round, it will be chosen one node on the chain path to transmit the aggregated data to the BS. To locate the closest neighbor node in PEGASIS, each node uses the signal strength to measure the distance of all neighbor nodes. Furthermore, it requires global information of the network known by each sensor node and this does not scale well where such global knowledge is not easy to obtain.

Since data generated in a sensor network is too much for end-user to process, so data aggregated is required. Power Efficient Data Gathering and Aggregation in 
Wireless Sensor Networks [9] is proposed. The framework of this proposal is a minimum spanning tree based network. It assumes the locations of all nodes are known by base station and the routing information is computed using Prim's minimum spanning tree algorithm where BS is the root. In this proposal, each round selects the minimum weighted edge from a vertex in the tree to a vertex not in the tree, and adds that edge to the tree. Sensor nodes will transmit the sensed data to BS via the routing path that is constructed before and it achieves a minimum energy consuming system. Nevertheless, the intermediate nodes will consume their energy quickly. And Hierarchy-Based Anycast Routing (HAR) Protocol for Wireless Sensor Networks [6] is proposed. In this scheme, BS constructs hierarchical tree by finding its child nodes by sending packets (such like CREQ, CREP, CACP, PREQ) and discover their own child nodes in turn and so on. The drawback is sending and receiving too many packets will expense much energy of the network.

In this paper, we propose a hierarchy-based multipath routing protocol (HMRP). In the HMRP scheme, the sensor network is constructed as a layered network at first. Based on the layered, sensor nodes will find their candidate parents and transmit the aggregated data to the BS. By way of those candidate parents, the sensor node will has multipath to reply the data. In HMRP, sensor node can switch the routing path to their candidate parents by turns. Our design can distribute the energy cost in the network more efficiently and prolong the network lifetime.

\section{HMRP: Hierarchy-Based Multipath Routing Protocol}

HMRP forms hierarchical relations by using a Layer Packet, where nodes can make autonomous relationship without any centralized control. Our goal is to design a hierarchy-based multipath routing algorithm such that when a node may belong to a certain number of parents, it can choose different parent to forward packet every time.

\subsection{Layer Packet Format}

The format of the layer packet used in HMRP is shown in Fig. 1. It consists of the HopCount, SourceID and SinkID fields. The HopCount field is the number of nodes between sink node and destination node. The nodes that can receive the radio signal of sink are defined as one-hop nodes. SourceID, the ID of the node that layer packet come from, and due to the HMRP support multiple sink, so the SinkID will indicate which one broadcast the layer packet. The number of bytes for each field is best to determine by the application designer. In this paper, we assume that the identifier of sensor nodes is determined a priori.



Fig. 1. Layer packet format in HMRP 


\subsection{Network Construction Procedures}

The main activities in this phase are hierarchy setup, candidate parents' selection, routing path formation, and information table creation for each node. During each setup phase, we define the hop value of sink is zero, and other sensor nodes are $\infty$. The base station first increase the HopCount field by one and broadcasts the layer packet to construct the whole network hop level. During the $T_{\text {lavertime }}$, if sensor node receives many layer packets will compare the HopCount field to choose a less one. And it stores the HopCount, SourceID and SinkID fields of layer packet into HopValue, CandidateParents and SinkID fields of its Node Information Table (NIT).

In Fig. 2, when sensor node 1 receives the layer packets (may come from different sink nodes or other nodes) will choose a node with the less value of HopCount to be its parent. And it will record all this information into NIT. After that, node 1 increase the HopCount field of layer packet by one and broadcast again. At the same time, node 4 will do the similar motion like node 1 . Node 6 received two layer packets from node 1 and 4 . Hence, its candidate parents are node 1 and 4, the layer packet come from sink A and node 6 is at 2 hop distance from sink A. Every node keeps flooding the layer packet until the network is constructed.

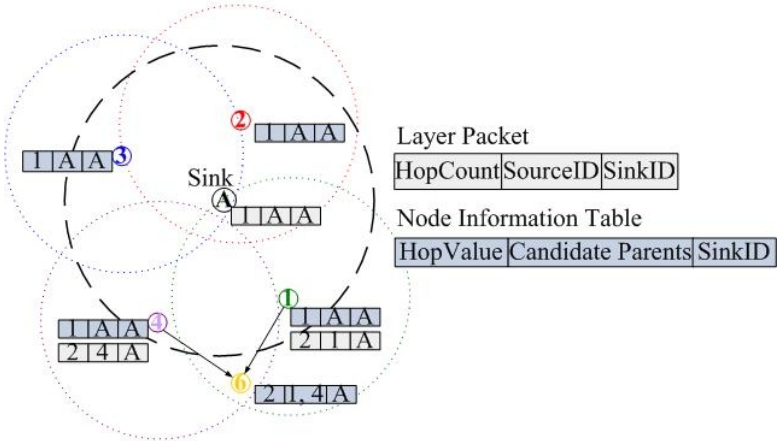

Fig. 2. Network construction flooding

\subsection{Data Transmission Phase (DTP)}

After finish the first phase, the network will be constructed like Fig. 3. Sensor nodes start disseminating the sensed data to the BS via the parent nodes. By using Round Robin method in the CandidateParents field of NIT, when sensor node $\mathrm{x}$ wants to disseminate data packet to sink, it will choose a parent $\mathrm{p}$ from CandidatePartents field by turns. And transfer data packet to $\mathrm{p}$, after $\mathrm{p}$ received this data packet will reply an Acknowledge (ACK) packet to ensure the transmission is successful. If there is no ACK reply from $\mathrm{p}$ after a Tconfirm time (this time is very short), node $\mathrm{x}$ will remove $\mathrm{p}$ from its NIT and transfer the data packet to next candidate parent. 




Fig. 3. Network construction result

For example: in Fig. 4, node 56 has five candidate parents, 20, 38, 39, 37 and 49. It will choose a parent from CandidateParents field sequent. At first time, node 56 disseminates data packet to parent node 20. If node 20 replies an ACK packet, the NIT of node 56 will move the node 20 to last position of CandidateParents field. Otherwise, node 20 will be deleted from the NIT, because its energy may run out or it is broken. Each node does the same motion like node 56 until the data packet reach the sink node.

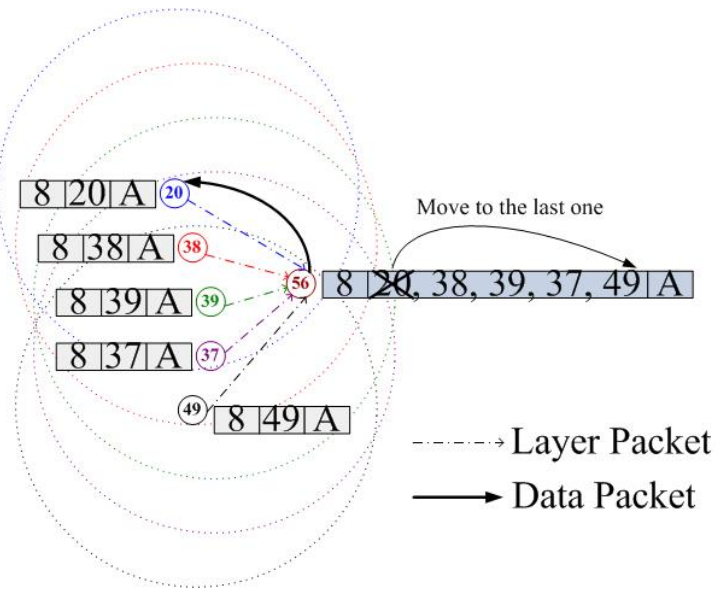

Fig. 4. Data dissemination

Relying on this manner, there are many paths can forward data packet to sink. And sensor node uses different path every time can extend the lifetime of network system. 


\subsection{HMRP Advantages}

HMRP employs hierarchical concept to construct whole sensor network. Each sensor node (involve sink node) only broadcast the layer packet once and maintain its own NIT. When sensor node disseminates data packet, it only need to know which parent node to transfer, don't need to maintain the whole path information. This can reduce the overhead of sensor node. Although HMRP has to computing some information to record in the NIT of sensor node, but the energy expense is less than transmit and receive. Furthermore, HMRP support multipath data forwarding, not use the fixed path. So the energy consume will be distributed and the lifetime of network will be prolonged. Finally, HMRP can allow multiple sink nodes.

\section{Simulation Results}

In our analysis, we use the first order radio model discussed in $[2,8]$. The transmit and receive energy costs for the transfer of a k-bit data message between two nodes separated by a distance of $r$ meters are given by Eqs. 1 and 2, respectively.

$$
\begin{gathered}
E_{T}(k, r)=E_{T x} k+E_{\text {amp }}(r) k \\
E_{R}(k)=E_{R x} k
\end{gathered}
$$

Where $\mathrm{E}_{\mathrm{T}}(\mathrm{k}, \mathrm{r})$ in Eqs. 1 denotes the total energy dissipated in the transmitter of the source node while in Eqs. 2, $\mathrm{E}_{\mathrm{R}}(\mathrm{k})$ represents the energy cost incurred in the receiver of the destination node. The parameters $\mathrm{E}_{\mathrm{Tx}}$ and $\mathrm{E}_{\mathrm{Rx}}$ are per bit energy dissipation for transmission and reception. $E_{\text {amp }}(r)$ is the energy required by the transmit amplifier to maintain an acceptable radio in order to transfer data message reliably. We use the free-space propagation model and the transmit amplifier $\mathrm{E}_{\mathrm{amp}}(\mathrm{r})$ is given by Eqs. 3 .

$$
E_{\text {amp }}(r)=\varepsilon_{F S} r^{2}
$$

Where $\varepsilon F S$ denote transmit amplifier parameter. We assumed the same set of parameters in $[2,8]$ for all experiments throughout this paper: $\mathrm{E}_{\mathrm{Tx}}=\mathrm{E}_{\mathrm{Rx}}=50 \mathrm{~nJ} / \mathrm{bit}, \varepsilon_{\mathrm{FS}}$ $=10 \mathrm{pJ} / \mathrm{b} / \mathrm{m} 2$ and the energy cost for the data aggregation is set as $E_{\mathrm{DA}}=5$ $\mathrm{nJ} / \mathrm{b} / \mathrm{message}$.

To evaluate the performance of HMRP, we use the $\mathrm{C}++$ language to run a number of simulations described in the section. We compare the performance with other cluster-based routing protocols such as LEACH, PEGASIS, HAR and PEDAP. The aim is to measure system lifetime and average energy dissipation. We generate networks of $100 \mathrm{~m} \times 100 \mathrm{~m}$ that having 500 nodes and we simulate with different topologies. Furthermore, the initial battery of each node is $2 \mathrm{~J}$ and the number of data frames transmitted for each round is set at 40 . In addition, we fix the message size at 500 bytes in all simulations and assume each sensor node has ability to transmit to BS directly.

Fig. 5 shows the system lifetime of those protocols. HMRP has a good lifetime improvement to others. In addition, we assume the system lifetime is defined as the number of rounds for which 75 percent of the nodes still alive. HMRP has improved the lifetime of other protocols: 200\% of LEACH, $8 \%$ of PEGASIS, 5\% of HAR and $14 \%$ of PEDAP. The improvement gained through HMRP is further exemplified by the average energy dissipation graph in Fig 6. HMRP performs energy consumed 


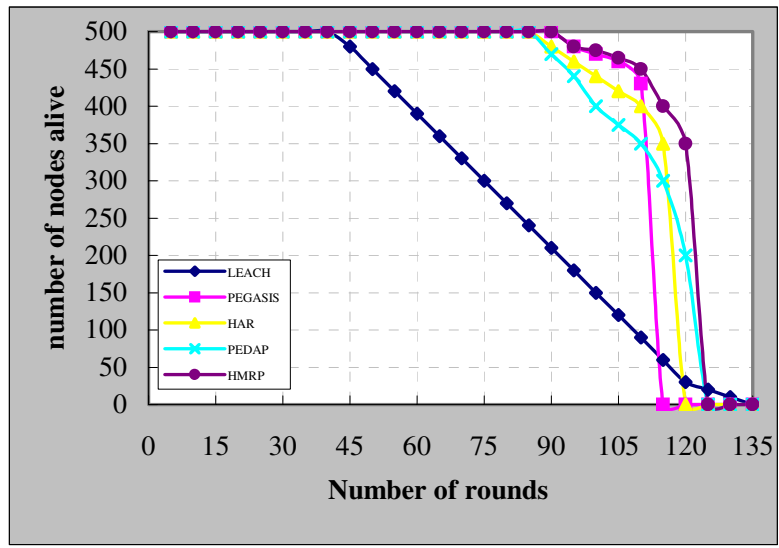

Fig. 5. A comparison of HMRP's system lifetime with other protocols

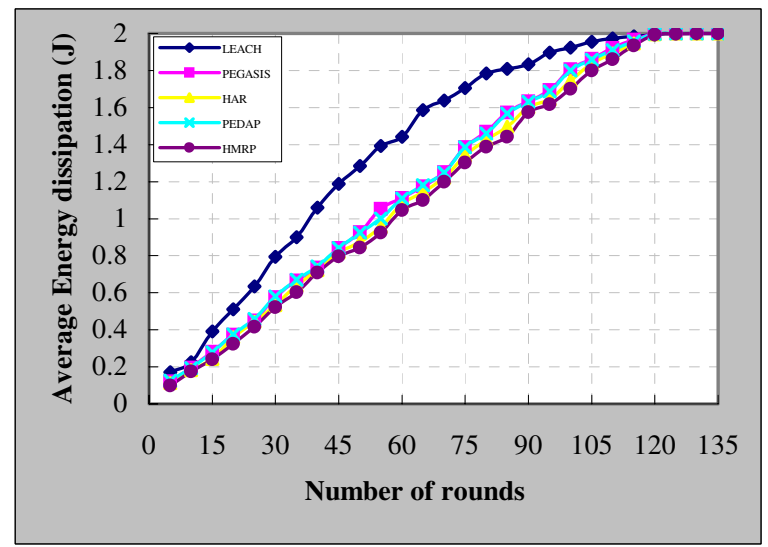

Fig. 6. A comparison of HMRP's average energy dissipation with other protocols

more efficiently in this plot. On average, HMRP displays a reduction in energy consumption of $35 \%$ over LEACH. This is because all cluster heads in LEACH transmit data to the BS directly. However, others alleviate this problem by having only one cluster head node forward the data to the BS. Nevertheless, HMRP still outperform PEGASIS $8 \%$ since the distance of neighbors, and HMRP outperforms HAR and PEDAP by $4 \%$ and $7 \%$, respectively. Because HMRP spent less cost of energy to construct the hierarchy-structure.

\section{Conclusions}

Energy recourse is limit and is the most important issue to sensor networks. Distributing the load to the nodes has a great impact on system lifetime. In this paper, we pro- 
pose a hierarchy-based multipath routing protocol and our main idea is minimizing the path loading of the system by distributing the energy consumption among the nodes. In HMRP, sensor nodes do not to maintain the information of the whole path and they just keep their NITs. We show through the simulation results, HMRP has a better performance then LEACH, PEGASIS, HAR and PEDAP. In addition, it is worth to note that HMRP also supports to multiple base stations (or Sink nodes).

\section{References}

1. Lindsey, S.; Raghavendra, C.S. "PEGASIS: Power Efficient Gathering in Sensor Information System", Aerospace Conference Proceedings, IEEE, pp. 1125-1130, vol 3, 2002.

2. Heinzelman, W.R.;Chandrakasan, .;Balakrishnan, H. "Energy-efficient communication protocol for wireless microsensor networks", System Sciences, 2000. Proceedings of the 33rd Annual Hawaii International Conference, pp. 3005-3014, 2000.

3. Shih-Chang Huang; Rong-Hong Jan. "Energy-Aware,Load Balanced Routing Schemes for Sensor Networks", Parallel and Distributed Systems, 2004. ICPADS 2004. Proceedings. pp. 419-425, 2004.

4. Qiangfeng Jiang; Manivannan, D. "Routing protocols for sensor networks", Consumer Communications and Networking Conference, 2004. CCNC 2004. First IEEE, pp. 93-98, 2004.

5. Al-Karaki, J.N.; Kamal, A.E. "Routing techniques in wireless sensor networks: a survey", Wireless Communications, IEEE, Issue: 6, pp. 6-28. Volume: 11, Dec. 2004.

6. Thepvilojanapong, N.; Tobe, Y.; Sezaki, K. "HAR: Hierarchy-Based Anycast Routing Protocol for Wireless Sensor Networks", Applications and the Internet, 2005. Proceedings. pp. 204-212, 2005.

7. Muruganathan, S.D.; Ma, D.C.F.; Bhasin, R.I.; Fapojuwo, A.O. "A Centralized EnergyEfficient Routing Protocol for Wireless Sensor Networks", communications Magazine, Issue 3, pp. s8-13, IEEE Volume 43, 2005.

8. W. B. Heinzelman, A. P. Chandrakasan, and H. Balakrishnan, "An Application-Specific Protocol Architecture for Wireless Microsensor Networks", IEEE Transactions on Wireless Communications, pp. 660-670, vol 1, no. 4, 2002.

9. Hüseyin Özgür Tan and Ibrahim Körpeo lu, "Power Efficient Data Gathering and Aggregation in Wireless Sensor Networks", SPECIAL ISSUE: Special section on sensor network technology and sensor data management, pp. 66-71, ACM SIGMOD Record, Volume 32, Issue 4, 2003. 